\title{
Influence de l'échantillonnage des communautés macrobenthiques sur l'évaluation de la qualité biologique d'un cours d'eau
}

\author{
J.-N. Beisel 1 \\ P. Usseglio-Polatera ${ }^{1,2}$ \\ S. Thomas 1 \\ J.-C. Moreteau1
}

Mots-clés : échantillonnage, macroinvertébrés, I.B.G.N., indices de structure, qualité biologique, rivières.

En rivière, le cumul d'échantillons prélevés sur plusieurs mésohabitats fournit généralement une image de la communauté macrobenthique d'une station, chaque mésohabitat étant défini comme la combinaison d'un type de substrat et d'une gamme de vitesse de courant. Des simulations ont permis d'étudier la variabilité de l'image de la communauté d'une station où 8 mésohabitats seraient pris en compte alors que 11 sont réellement disponibles. Les résultats sont replacés dans le cadre de l'utilisation de l'Indice Biologique Global Normalisé (IBGN).

Les indices de structure taxinomique sont extrêmement variables suivant la combinaison considérée. La richesse taxinomique est l'indice le plus stable si on le rapporte à sa gamme de variation. A l'opposé, l'abondance varie énormément. Les indices de diversité/dominance occupent une place intermédiaire. Plus un indice de structure intègre les abondances des taxons, plus il dépend des mésohabitats échantillonnés. La richesse taxinomique est intégrée à l'IBGN sous forme de classes, ce qui atténue l'effet de sa variabilité. Tout en respectant le protocole d'échantillonnage, l'incertitude sur la note IBGN peut atteindre 3 points. Des solutions sont envisagées pour optimiser les méthodes de suivi de la qualité biologique d'un cours d'eau.

\section{Effects of sampling on benthic macroinvertebrate assemblages in assessment of biological quality of running water}

Keywords : sampling, macroinvertebrates, I.B.G.N., structure indices, biological quality, streams

In running waters, a picture of the macroinvertebrate community of a station is generally obtained by adding individuals sampled in different mesohabitats. Mesohabitats can be defined as combinations of a substrate type and a range of current speed. Simulations allowed us to study the variation in the community picture of a station when 8 mesohabitats were taken into account although eleven were sampled. Results were replaced in the framework of the use of the Indice Biologique Global Normalise (IBGN).

Taxonomic structure described with indices varied greatly with the combination of mesohabitats sampled. Taxonomic richness was the more stable index considering the potential range of this index. In contrast, abundance was the more variable index. Between these extremes, diversity or dominance indices had an intermediate range of variability. The variability of a structure index seemed to increase when this index integrates taxa abundances, which depend on mesohabitats sampled. Taxonomic richness is integrated in IBGN in the form of classes; this expression reduces the effect of richness variability. Even if the sampling protocol was respected, the uncertainty on the IBGN can reach 3 units. Solutions have been considered to optimize the assessment of running water biological quality.

\section{Introduction}

En rivière, les protocoles d'échantillonnage d'une station, qu'ils soient stratifiés ou orientés, s'appliquent

1. UPRES «Ecotoxicité, Biodiversité et Santé Environnementale» (E.B.S.E.), Université de Metz, UFR Sciences, Ile du Saulcy, F57045 Metz Cedex 01, France.

2 UPRESA CNRS 5023 «Ecologie des Eaux Douces et des Grands Fleuves», Université Lyon I, F-69 622 Villeurbanne Cedex, France. généralement à prélever un large éventail de mésohabitats. Un mésohabitat peut être défini comme la combinaison de deux paramètres physiques du milieu : le substrat et la vitesse de courant. L'image de la communauté macrobenthique stationnelle est alors obtenue par le cumul des listes faunistiques des échantillons prélevés sur différents mésohabitats.

Chaque mésohabitat a des qualités intrinsèques susceptibles d'influencer sa communauté de macroinvertébrés, tant en terme de composition qu'en terme d'or- 
ganisation. Dans ces conditions, la nature des endroits prélevés influence forcément l'image restituée du peuplement stationnel. Or, sur une station diversifiée en termes d'habitats, il est peu probable que deux hydrobiologistes prélèvent strictement la même combinaison d'habitats, et obtiennent par conséquent la même image de la communauté d'invertébrés autochtone.

L'objet de cet article est d'évaluer la variabilité des résultats faunistiques susceptibles d'être obtenus à l'échelle d'une station (1) si l'on fixe à huit le nombre de mésohabitats à prélever, et (2), compte tenu des différentes combinaisons de mésohabitats réalisables pour obtenir la liste faunistique stationnelle. Les résultats sont replacés dans le cadre de l'utilisation de l'Indice Biologique Global Normalisé (noté IBGN), indice biotique le plus utilisé en France et qui fait l'objet d'une norme française homologuée (A.F.N.O.R. 1992).

\section{Matériel et méthodes}

\subsection{Sites d'étude}

L'échantillonnage a été réalisé au printemps et en automne de 1993 et 1994 sur 7 stations situées sur des cours d'eau lorrains d'ordre 1 à 4 . Le choix des stations se devait de réunir un large éventail de situations environnementales pouvant engendrer une grande variété de réponses faunistiques. Il a également été dicté (1) par le besoin de réaliser une cartographie des mésohabitats du lit mineur, (2) par la nécessité de pouvoir prélever n'importe lequel des mésohabitats identifiés, (3) par la volonté de ne travailler que sur des tronçons de rivière exempts de problèmes importants de qualité d'eau.

Les stations étudiées se situent sur le haut bassinversant de la Meuse, sur le bassin-versant de la Mortagne (affluent de la Meurthe), et sur le bassin-versant de la Bruche (affluent de l'Ill). Les gammes de variation des variables mesurées durant les prélèvements hydrobiologiques sont présentées en figure 1 . Un descriptif détaillé des stations peut être trouvé dans Beisel (1996).

Le nombre de mésohabitats disponibles peut varier de 9 à 12 sur une même station entre le printemps et l'automne, ou entre 1993 et 1994. Par souci d'homogénéité, nous n'avons retenu pour cette analyse que 11 station/date ayant comme point commun la présence de 11 mésohabitats exactement, chacun d'eux ayant été échantillonnés une fois. L'objectif de ce travail nécessite en effet des stations diversifiées en mésohabitats pour que se pose le problème du choix des endroits à prélever. Le terme «station» a été conservé pour dé- signer la combinaison d'une station (parmi les 7 prospectées), et d'une des 4 campagnes de prélèvements hydrobiologiques.

\subsection{Stratégie d'échantillonnage}

De façon à pouvoir repérer les mésohabitats à échantillonner, la mosaïque des substrats est cartographiée avec précision au début de chaque campagne d'échantillonnage. Le mésohabitat a été défini comme la combinaison d'un type de substrat (parmi 39 modalités possibles, inspirés d'un découpage des substrats proposé par Wasson et al. 1981) et d'une gamme de vitesse de courant (parmi 5 modalités, qui correspondent strictement aux classes de la norme IBGN, A.F.N.O.R. 1992). L'objectif de notre protocole est d'échantillonner le plus large éventail possible de mésohabitats (protocole détaillé dans Beisel 1996), sur chacune des stations retenues.

Onze mésohabitats ont été prélevés dans chacune des 11 stations. Les macroinvertébrés ont été échantillonnés à l'aide d'un filet de type Surber modifié de $1 / 20^{\text {ème }}$ de $\mathrm{m}^{2}$ avec un vide de maille de $0,5 \mathrm{~mm}$. Le substrat, complètement prélevé dans le filet, est formolé à $4 \%$ pour être trié ultérieurement. Les macroinvertébrés sont extraits des différents supports et comptés.

Les déterminations sont poussées au plus loin mais par souci d'homogénéité, les résultats présentés correspondent à des listes faunistiques identifiées au niveau taxinomique de la famille pour tous les taxons, à l'exception des oligochètes et des hydracariens.

\subsection{Méthode de simulation et analyse}

Pour chaque station, nous avons simulé toutes les images de communautés stationnelles susceptibles d'être obtenues par cumul des listes faunistiques de 8 prélèvements parmi les 11 disponibles (165 possibilités : Fig. 2). Le nombre de listes faunistiques cumulées a été fixé à 8 de manière à respecter le nombre d'échantillons préconisé dans la norme IBGN (A.F.N.O.R. 1992),

Ces listes ont été comparées en terme d'IBGN et d'indices de structure largement utilisés dans le diagnostic ou le suivi de la qualité biologique d'un milieu. Les indices de structure (Fig. 2) n'ayant pas les mêmes ordres de grandeur, ils ont été comparés sur la base de leurs coefficients de variation (notés CV), calculés sur les résultats des 11 stations :

$$
\mathrm{CV}(\%)=\frac{\text { Ecart }- \text { type }}{\text { Moyenne }} \times 100
$$




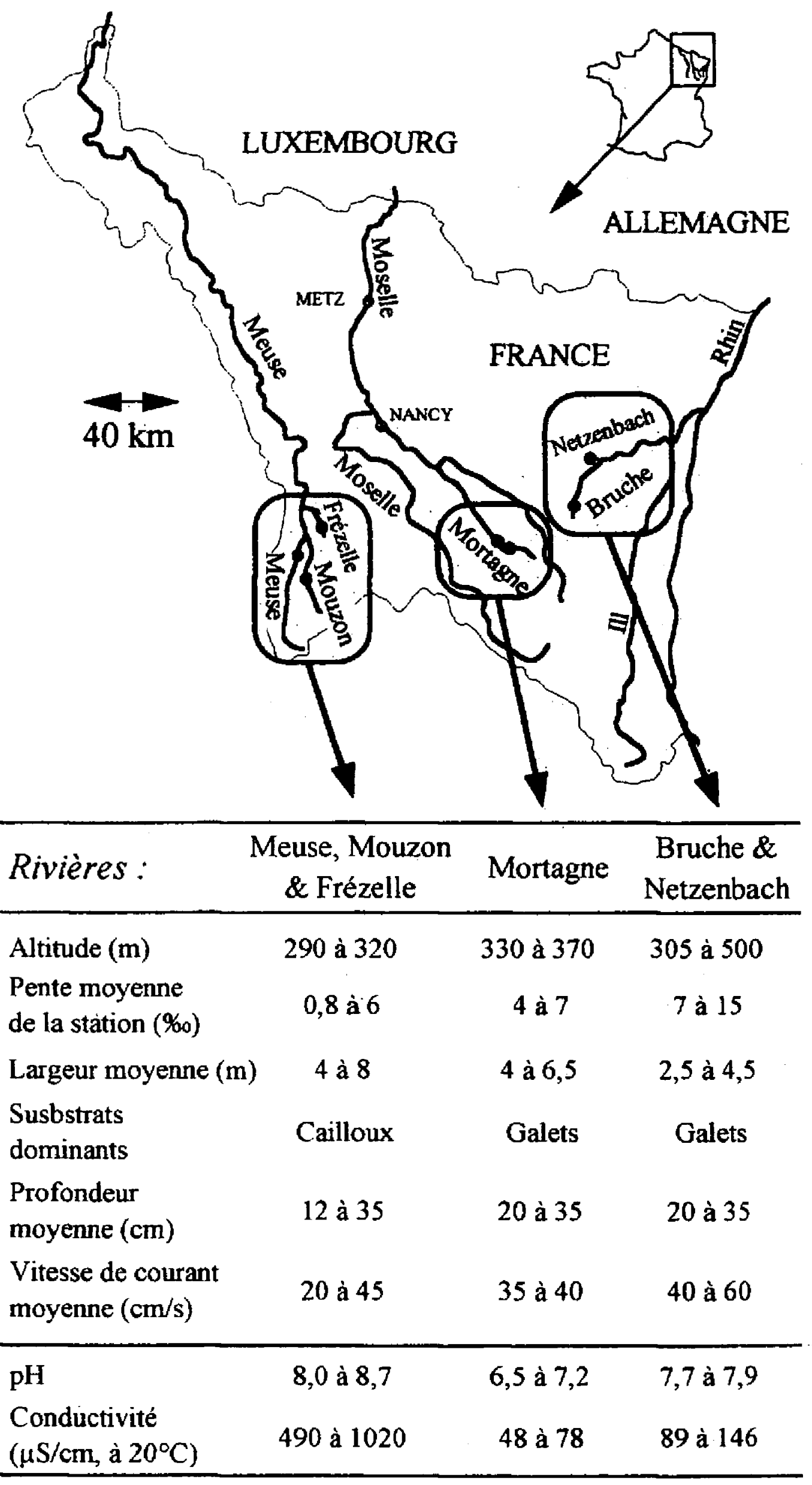

Fig. 1. Cartes des stations étudiées. Le tableau présente les gammes de variations de caractéristiques mésologiques lors des campagnes d'échantillonnage.

Fig. 1. Map of the sampling sites. The table presents the range of mesological characteristics on the sampling dates. 


\section{(A) Tableau faunistique}

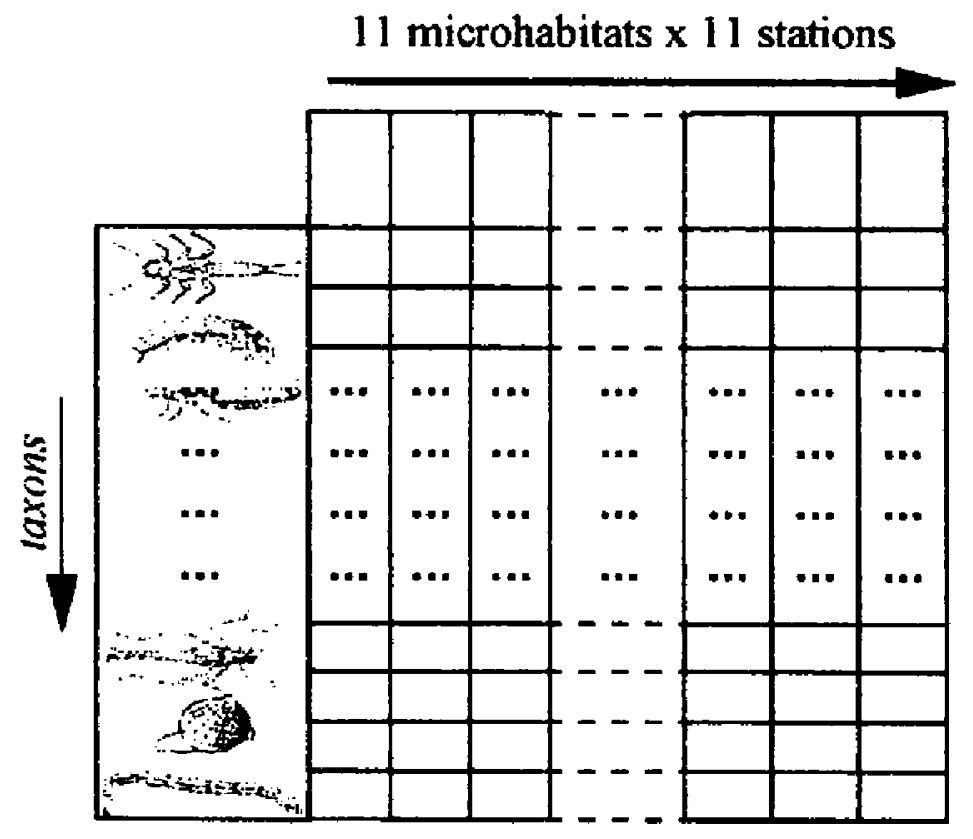

B Simulations : 8 listes faunistiques de microhabitats cumulées pour obtenir une image de la communauté stationnelle

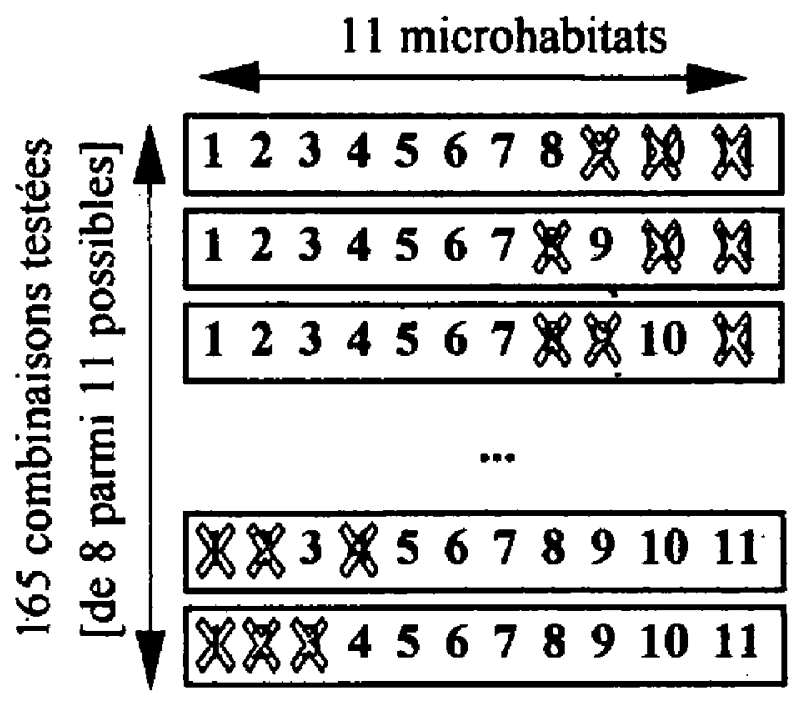

C) Pour chacune des 11 stations, comparaison des 165 communautés obtenues avec:

\section{$\Rightarrow 5$ indices de structure faunistique}

\begin{tabular}{ccc}
\hline Nom & Sigle & Formule \\
\hline 1. Richesse & $\mathrm{S}$ & Nombre de taxons \\
2. Abondance & $\mathrm{Q}$ & $\begin{array}{c}\text { Nombre total d'individus } \\
\sum_{\mathrm{i}=1}^{\mathrm{S}} \mathrm{q}_{\mathrm{i}}\end{array}$ \\
$\begin{array}{c}\text { 3. Diversité } \\
\text { (Shannon-Weaver 1963) }\end{array}$ & $\mathrm{H}^{\prime}$ & $-\sum_{\mathrm{i}=1}^{\mathrm{S}}\left(\frac{\mathrm{q}_{\mathrm{i}}}{\mathrm{Q}}\right) \log _{2}\left(\frac{\mathrm{q}_{\mathrm{i}}}{\mathrm{Q}}\right)$ \\
$\begin{array}{c}\text { 4. Equitabilité } \\
\text { (Hurlbert 1971) }\end{array}$ & $\mathrm{e}$ & $\frac{\mathrm{H}^{\prime}-\mathrm{H}_{\min }}{\mathrm{H}_{\max }-\mathrm{H}_{\min }}$ \\
$\begin{array}{c}\text { 5. Dominance } \\
\text { (Simpson 1949) }\end{array}$ & $\lambda$ & $\sum_{\mathrm{i}=1}^{\mathrm{s}} \mathrm{q}_{\mathrm{i}}\left(\mathrm{q}_{\mathrm{i}}-1\right)$ \\
\hline
\end{tabular}

$q_{i}=$ abondance du toxon $i$

$H_{\text {min }}^{\prime}=$ diversite minimale, $H_{\max }^{\prime}=$ diversité maximale

\section{$\Rightarrow 1$ indice biotique : I'Indice Biologique Global Normalisé (IBGN, AFNOR 1992)}

Fig. 2. Méthode de simulation et analyse des communautés macrobenthiques simulées. (A) Le tableau faunistique analysé correspond à onze stations où onze mésohabitats ont été échantillonnés. (B) Pour chaque station, les 165 combinaisons de 8 mésohabitats parmi les 11 disponibles sont testées. (C) Les listes faunistiques sont comparées à l'aide de cinq indices de structure et un indice biotique.

Fig. 2. Simulation procedure and analysis of simulated macroinvertebrate communities. (A) The faunistic array corresponds to eleven stations where eleven mesohabitats were sampled. (B) For each station, 165 combinations of eight mesohabitats among eleven available were tested. (C) Simulated communities were compared both with five structure indices and a biotic index. 


\section{Résultats}

\subsection{Variabilité des IBGN}

L'IBGN intègre dans son calcul la richesse taxinomique et le groupe faunistique indicateur (GFI) qui attribue à certains taxons un niveau de sensibilité aux perturbations. Le GFI le plus élevé d'un taxon présent avec au moins 3 ou 10 individus, selon les cas, est retenu pour déterminer la note IBGN (A.F.N.O.R. 1992). Des variations dans l'estimation de la richesse taxinomique et/ou du GFI ont par conséquent une influence forte sur l'estimation de l'IBGN (Fig. 3). La richesse taxinomique peut varier de $+4(27$ à 31$)$ à + 11 (26 à 37) taxons sur une même station si l'on considère l'écart entre la combinaison de mésohabitats la plus pauvre et la plus riche.

Quatre stations sur onze conservent la même valeur de GFI quelle que soit la combinaison de mésohabitats considérées. Sur les autres stations, le GFI varie de 1 à 3 unités. Dans le cas unique où l'écart est de 3 (rivière Mouzon), le groupe indicateur le plus sensible (Ephemeroptera Leptophlebiidae) n'est présent que sur un mésohabitat et avec un effectif de 3 individus. La prise en compte ou non de ce mésohabitat dans la combinaison stationnelle peut faire chuter le GFI de 7 à 4 . Même si l'on considère les individus d'un même GFI comme des équivalents écologiques à comptabiliser ensemble, comme cela a été proposé par le passé (Agences de l'eau 1993), la non prise en compte de l'échantillon avec des Leptophlebiidae fait chuter le GFI à 4.

Les écarts de note IBGN pour une même station sont au minimum de 1 point, et au maximum de 5 points, ce qui est important. Ce dernier cas, sur la rivière Mouzon, correspond à la non-prise en compte d'un mésohabitat qui fait chuter le GFI de 7 à 4 . La note IBGN varie alors de 17 à 12/20. Toutes les combinaisons de nos simulations de 8 mésohabitats parmi les 11 disponibles ne respectent cependant pas strictement le protocole d'échantillonnage préconisé par la norme. Si l'on suit le protocole il devient en effet obligatoire de prélever le mésohabitat qui héberge le plus fort GFI puisqu'il est le seul qui correspond à la combinaison [cailloux $\mathrm{x}$ vitesse de courant $<5 \mathrm{~cm} / \mathrm{s}$ ] (Fig. 4). Cependant, des choix subsistent parmi des prélèvements correspondant à une même combinaison substrat $x$ vitesse de courant. Si l'on ne considère que les 12 combinaisons qui respectent strictement la norme, la note IBGN ne varie plus que de 15 à 17. Sans que le protocole d'échantillonnage ne soit outrepassé, l'incertitude sur la note IBGN est donc encore de 2 points. Sur d'autres stations, cette incertitude peut atteindre 3 points.

\subsection{Variabilité des indices de structure taxino- mique}

La répartition des valeurs d'un indice de structure suit généralement une loi normale (Fig. 5). Cependant, dans 4 cas sur 11 , la distribution des résultats de richesse taxinomique est bimodale. Il s'agit de situations où un mésohabitat sur les 11 échantillonnés est occupé par des taxons non rencontrés ailleurs (entre 4 et 7 taxons). La prise en compte ou non de ce mésohabitat fournit alors des valeurs de richesse avec 4 à 6 points de différence.

D'une manière générale la variabilité des indices descripteurs de la structure taxinomique est importante. Dans le cas illustré Fig. 5, d'une des 11 stations, les valeurs extrêmes des indices en fonction des mésohabitats considérés montrent une grande amplitude, l'écart entre extremums atteignant un facteur de 1,8 pour l'abondance numérique. L'interprétation de la structure d'une communauté peut donc être notablement différente suivant la combinaison de mésohabitats retenus pour établir la liste faunistique de la station.

Les CV moyens permettent de comparer la variabilité d'indices qui n'ont pas les mêmes échelles de variation (Fig. 6). La richesse taxinomique a la dispersion la plus faible sur l'ensemble des différentes combinaisons. La diversité de Shannon-Weaver et l'équitabilité de Hurlbert sont ensuite les paramètres les plus stables. L'abondance numérique, et dans une moindre mesure l'indice de dominance de Simpson, ont une plus grande variabilité. D'une manière générale, il semblerait qu'un indice de structure taxinomique est d'autant plus variable qu'il est plus sensible aux abondances des taxons. Comparativement à ces indices l'IBGN apparaît comme un descripteur faunistique avec une faible variabilité (Fig. 6).

\section{Discussion et conclusion}

\subsection{Variabilité des indices de structure}

Les indices décrivant la structure d'un peuplement sont couramment utilisés pour décrire l'état d'une communauté d'invertébrés benthiques.

La richesse taxinomique semble le paramètre le plus fiable pour des comparaisons inter-stationnelles, sous réserve que les échantillons aient toujours la même taille (Ludwig \& Reynolds 1988). Lors d'une étude de la variabilité des communautés macrobenthiques de cailloux, Downes et al. (1993) ont également montré que la richesse variait peu, quelle que soit l'échelle spatiale considérée : habitat, radier, ou tronçon de rivière. 

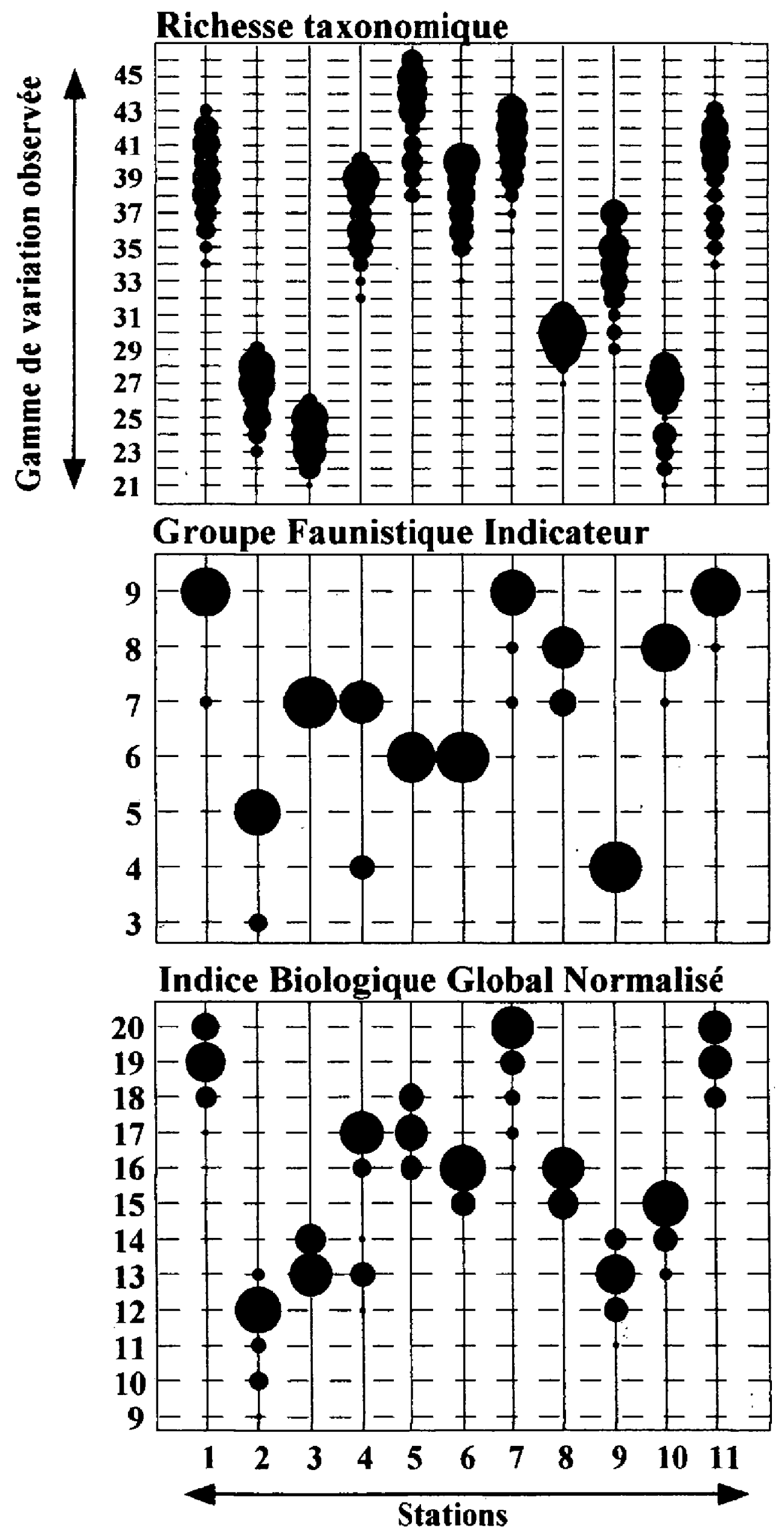

Fig. 3. Variabilité de la richesse, du groupe faunistique indicateur et de l'BGN, pour chacune des 11 stations étudiées. La taille des points est proportionnelle à la fréquence d'obtention d'un résultat au sein des 165 communautés obtenues par simulations sur une station.

Fig. 3. Variation of taxonomic richness, faunistic indicator group, and IBGN; for each of the eleven stations studied. The circle area is proportional to the frequency of the different values obtained with the 165 simulated communities. 


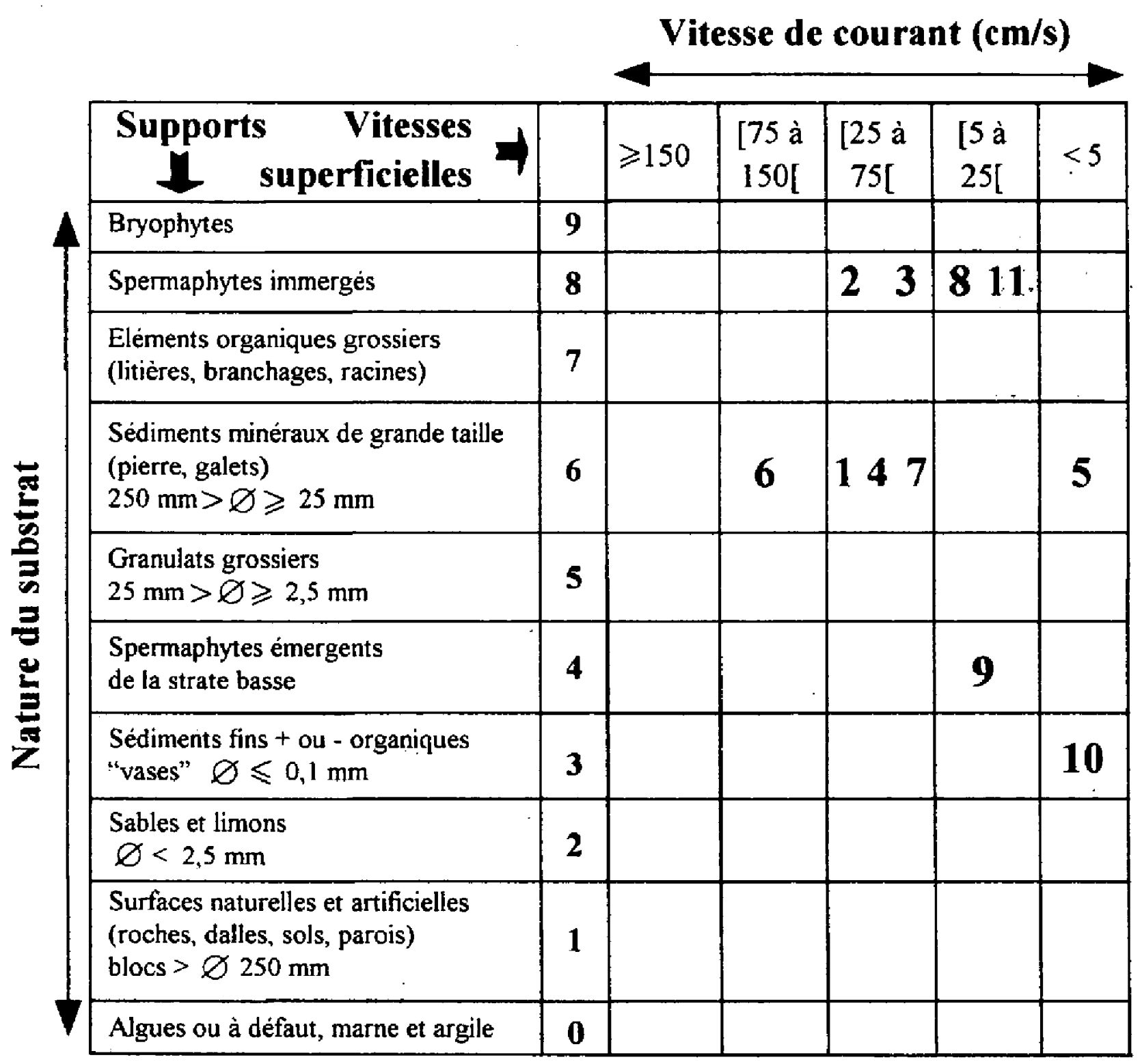

Fig. 4. Illustration de la combinaison de 11 mésohabitats échantillonnés sur une station $\left(N^{\circ} 4\right.$ sur la Fig. 3 , rivière Mouzon). Les mésohabitats, numérotés de 1 à 11, sont replacés dans le cadre du protocole d'échantillonnage de 1'IBGN (A.F.N.O.R. 1992).

Fig. 4. An example of a combination of mesohabitats sampled on a station. ( $\mathrm{N}^{\circ} 4 \mathrm{Fig}$. 3). Mesohabitats (number from 1 to 11) are positioned in the framework of IBGN sampling protocol (A.F.N.O.R. 1992).

Lorsque l'on considère des combinaisons de 8 mésohabitats parmi 11 disponibles, les différences de richesse proviennent forcément d'invertébrés présents sur un très petit nombre de mésohabitats. Or, tous les mésohabitats ont un cortège de taxons de ce type dans les assemblages d'invertébrés qu'ils hébergent. Pour toute combinaison, il existerait donc une sorte de compensation des taxons absents dans un mésohabitat par ceux présents uniquement dans d'autres. La somme des listes faunistiques de «n» mésohabitats foumit par conséquent, suivant les combinaisons, une image différente du peuplement sur le plan de la composition mais relativement régulière ( $\mathrm{si}$ l'on considère son échelle de variation potentielle) pour ce qui concerne la richesse taxinomique.
A l'inverse de la richesse taxinomique, la valeur de l'abondance numérique dépendrait fortement des caractéristiques des mésohabitats échantillonnés. Downes et al. (1993) ont montré des différences significatives de densités même à de petites échelles d'observation comme, par exemple, pour différentes zones de cailloux d'un même radier.

Parmi les indices de diversité/dominance, l'indice de diversité de Shannon-Weaver et l'équitabilité ont une variabilité relative quasi-identique. L'indice de Shannon-Weaver est sensible aux abondances relatives des taxons moyennement représentés alors que l'indice de dominance de Simpson est très sensible aux abondances des taxons les mieux réprésentés (Peet 1974). 


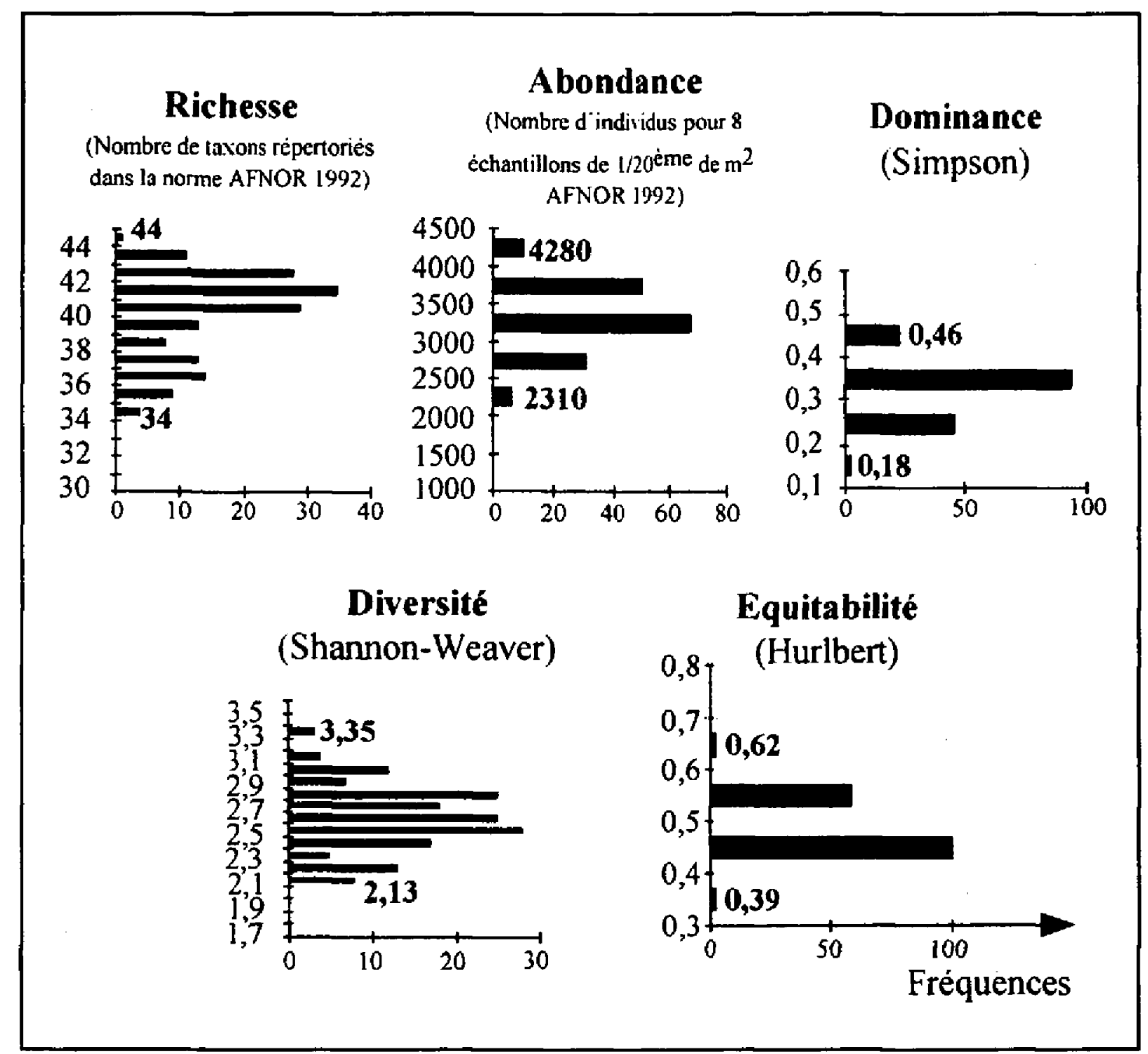

Fig. 5. Distribution de fréquence de cinq indices de structure calculés sur les 165 listes faunistiques simulées d'une des onze stations échantillonnées ( $\mathrm{N}^{\circ} 11$ sur la Fig. 3, rivière Netzenbach). Les chiffres a l' intérieur des graphiques représentent les valeurs extrêmes obtenues sur au moins une communauté simulée.

Fig. 5. Distribution of values calculated on 165 simulated faunistic lists of a station $\left(\mathrm{N}^{\circ} 11\right.$ on the Fig. 3, Netzenbach River), for five structure indices. Numbers inside the graph represent extreme values obtained for at least a simulated community.

La forte variabilité de ces deux indices est liée à la forte variabilité des abondances des taxons moyennement ou fortement représentés.

Les abondances relatives observées à l'échelle de la station dépendent de l'organisation des assemblages d'invertébrés de chacun des mésohabitats qui composent l'échantillon stationnel. Les taxons étant répartis de manière agrégative et $n$ 'ayant pas tous la même dynamique de population ni le même effectif, l'organisation des assemblages faunistiques de mésohabitats est extrêmement variable. Par conséquent, le cumul de listes faunistiques issues de milieux dominés par des taxons différents aboutit à une grande variété poten- tielle d'images de la communauté stationnelle, en terme de structure notamment. La répartition agrégative des populations macrobenthiques est à la base de la variabilité observée des indices de diversité/dominance et de l'abondance. D'où l'intérêt de ne pas se baser sur un seul type d'indice.

\subsection{Variabilité de l'IBGN}

L'IBGN peut varier de 1 à 3 points, en fonction des mésohabitats échantillonnés, sans que les standards de son protocole d'échantillonnage ne soient outrepassés. Ces différences nous paraissent importantes car elles peuvent modifier significativement le diagnostic de qualité biologique porté sur un tronçon de rivière. 


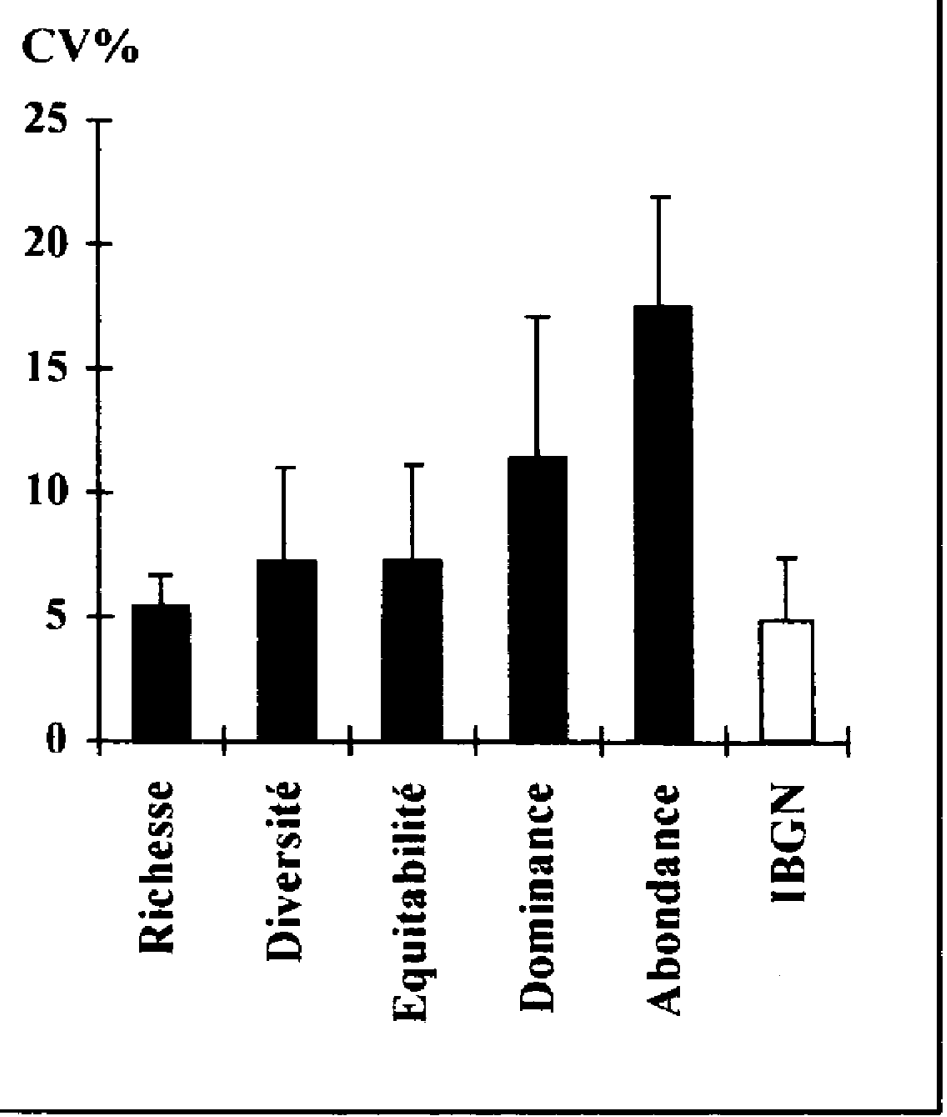

Fig. 6. Coefficients de variation moyens et écart-types par indice de structure et pour l'IBGN calculés sur les 11 stations.

Fig. 6. Mean and standard deviation of coefficients of variation for 5 structure indices and the IBGN calculated on 11 stations.

Dans le cas d'une station, le strict respect du protocole permet de diminuer significativement la variabilité de la note IBGN. Il serait cependant risqué de généraliser ce résultat qui n'est peut-être qu'un cas d'espèce.

Un avantage certain de l'IBGN est d'intégrer la valeur de la richesse taxinomique sous la forme de classes (= variété taxinomique). Cette transformation a pour effet d'atténuer l'incertitude sur la note IBGN due à la variabilité de la richesse. Il est également positif que ce soit l'indice de structure le moins sensible à la combinaison de mésohabitats échantillonnés qui soit intégré à l'IBGN, lui conférant ainsi une variabilité relative faible.

Par contre, des différences de groupe faunistique indicateur de 2 ou 3 niveaux nous paraissent importantes si l'on considère que 10 niveaux seulement sont possibles, de 0 (aucun groupe indicateur n'est présent) à 9 (Chloroperlidae, Perlidae, Perlodidae, Taeniopterygidae). Un tel écart provient également de la présence de certains taxons indicateurs dans un nombre souvent très limité de mésohabitats, avec des effectifs proches du nombre minimal d'individus nécessaire à leur prise en compte. Le système du groupe faunistique indicateur aurait probablement gagné à intégrer simultanément la présence de plusieurs taxons. Cette question a déjà été soulevée par le passé (Agences de 1'eau 1993); le principe en a été adopté par les britanniques, entre autres, pour le calcul du Biological Monitoring Working Party score system (BMWP) décrit par Armitage et al. (1983).

\subsection{Quelles solutions pour pallier cette variabilité ?}

L'influence de facteurs écologiques naturels sur les communautés macrobenthiques complique considérablement l'utilisation de celles-ci pour l'appréciation de la qualité biologique d'un cours d'eau.

Nos résultats concernent des stations où 11 mésohabitats ont été échantillonnés. Il est possible que sur des stations plus diversifiées en terme d'habitats, l'incertitude sur l'IBGN ou sur une observation biologique serait encore plus grande que ce que nous avons observé; alors que sur des stations plus pauvres en mésohabitats, l'incertitude serait plus faible.

Des propositions peuvent être faites pour améliorer le suivi de la qualité d'un milieu.

Une première étape serait d'optimiser l'aspect descriptif du protocole d'échantillonnage et de la station, ce que proposaient déjà Verneaux \& Tuffery (1967) dans le protocole de l'indice biotique. Pour Wasson et al. (1981), la description précise des habitats disponibles et/ou échantillonnés permettrait au moins de comparer des communautés macrobenthiques sur une base solide. Dans le même esprit, Armitage \& Pardo (1995) suggèrent de réaliser systématiquement une cartographie du fond de la station. Un inventaire des mésohabitats permettrait également de rapporter la note IBGN d'une station aux capacités d'accueil potentielles de cette station.

Par la suite, le diagnostic porté sur la qualité d'une station sera d'autant plus fiable qu'il sera basé sur une conjonction de conclusions biologiques. Les informations recueillies à l'échelle de la station gagneraient par exemple à être complétées par une exploitation rationnelle des assemblages macrobenthiques de certains mésohabitats. Cette démarche a été développée dans des pays de l'hémisphère sud, 'où l'utilisation unique de substrats caillouteux est devenue extrêmement populaire (Scrimgeour et al. 1993), du fait des avantages de ce type de protocole (Lake et al. 1985). La restriction de l'étude à l'analyse faunistique d'un habitat particulier ne doit cependant pas introduire systématiquement des erreurs susceptibles d'affecter les conclusions portées sur un milieu (Green 1979). L'étude ap- 
profondie des communautés en place sur un mésohabitat particulier serait plutôt à considérer comme une source potentielle d'informations nouvelles et complémentaires sur la qualité du milieu.

Sans modifier le protocole de la norme IBGN, il parait recommandé d'établir systématiquement 8 listes faunistiques séparées, correspondant aux 8 prélèvements obligatoires, plutôt qu'une seule liste où tous les échantillons sont cumulés. L'exploitation des caractéristiques des assemblages d'invertébrés de mésohabitats permettrait d'affiner les diagnostics portés sur un milieu. La mise en place de cette approche nous semble cependant nécessiter des études complémentaires selon trois axes : (1) les relations faune-milieu à l'échelle du mésohabitat, (2) l'impact d'une perturbation sur les assemblages de mésohabitats, (3) la variabilité des assemblages faunistiques au sein d'un même type de mésohabitats.

\section{Remerciements}

Nous remercions vivement J. Gagneur (Toulouse) et un référé anonyme pour leurs remarques et suggestions sur une version préliminaire du manuscrit.

\section{Travaux cités}

A.F.N.O.R. 1992. - Essais des eaux. Détermination de l'indice biologique global normalisé (I.B.G.N.). Norme bomologuée T 90 350:1-8.

Agences de l'eau 1993. — Etude bibliographique des méthodes biologiques d'évaluation de la qualité des eaux de surface continentales. Etude Inter-Agences $\mathrm{N}^{\circ} 35.3$ volumes.
Armitage P.D., Moss D., Wright J.F. \& Furse M.T. 1983. — The performance of a new biological water quality score system based on macroinvertebrates over wide range of unpolluted running-water sites. Water Research, $17: 333-347$.

Armitage P.D. \& Pardo I. 1995. - Impact assessment of regulation at the reach level using macroinvertebrate information from mesohabitats. Regul. Rivers, $10: 147-158$.

Beisel J.N. 1996. - Microrépartition des invertébrés benthiques en eau courante: caractéristiques des microhabitats et organisation de leurs peuplements. Thèse de Doctorat, Univ. Metz : $259 \mathrm{p}$.

Downes B.J., Lake P.S. \& Schreiber E.S.G. 1993. - Spatial variation in the distribution of stream invertebrates. Implication of patchiness for models of community organization. Freshwater Biol., $30: 119-132$.

Green R.H. 1979. - Sampling design and statistical methods for environmental biologists. Wiley \& Sons, New-York : 257 p.

Hurlbert S.H. 1971. - The nonconcept of species diversity: a critique and alternative parameters. Ecology, $52: 577-586$.

Lake P.S., Doeg T. \& Morton D.W. 1985. - The macroinvertebrate community of stones in an Australian upland stream. Verh. Int. Verein. Limnol, 22 : 2141-2147.

Ludwig J.A. \& Reynolds J.F. 1988. - Statistical ecology. A primer on methods and computing. Wiley \& Sons, New-York : $337 \mathrm{p}$.

Peet R.K. 1974. - The measurement of species diversity. Ann. Rev. Ecol. Syst., $5: 258-307$.

Scrimgeour G.J., Culp J.M. \& Glozier N.E. 1993. - An improved technique for sampling lotic invertebrates. Hydrobiologia, 254 : 65-71.

Shannon C.E. \& Weaver W. 1963. - The mathematical theory of communication. Univ. nlinois Press, Urbana : 117 p.

Simpson E.H. 1949. - Measurement of diversity. Nature, $163: 688$.

Verneaux J. \& Tuffery G. 1967. — Une méthode zoologique pratique de détermination de la qualité biologique des eaux courantes. Indices biotiques. Ann. Sci. Univ. Besançon, 3 : 79-89.

Wasson J.G., Dumont B. \& Trocherie F. 1981. — Protocole de description des habitats aquatiques et de prélèvements des invertébrés benthiques dans les cours d'eau. CEMAGREF Lyon : $18 \mathrm{p}$. 